\title{
Clumping Of Branches of Axillary Artery-A Case Study
}

\author{
Mohanty S.R ${ }^{1}$, Sar Mamata ${ }^{2}$ \\ ${ }^{1}$ (Department of anatomy, Kalinga institute of medical sciences, KIIT university, india ) \\ ${ }^{2}$ (Department of anatomy, V.S.S medical college, sambalpur university, india)
}

\begin{abstract}
The increasing use of invasive diagnostic and interventional procedures in cardiovascular diseases makes it important to study the vascular variations of upper limb. Awareness of the possible variations will reduce the risk of complications like bleeding during surgical procedures. .Axillary artery is the continuation of subclavian artery, at the outer border of the first rib and from the lower border of teres major it becomes the brachial artery. During its course it is divided into 3 parts by pectoralis minor muscle. Usually 6 branches arise from different parts of the artery. The present study was carried out in 30 cadavers (10 females and 20males) of the department of anatomy v.s.s medical college, burla and all the branches of axillary artery were studied. There are many reports showing variations in the branching pattern of axillary artery. However, in our study some unusual variations were found.
\end{abstract}

Keywords: Axillary artery, Clumping, Subscapular artery, Variation

\section{INTRODUCTION}

Axillary artery is the direct continuation of the subclavian artery at the outer border of the first rib. The course of the axillary artery is anatomically divided into three parts by the pectoralis minor muscle. The first begins at the lateral border of the first rib and extends to the superomedial border of the pectoralis minor muscle. The first part is enclosed within the axillary sheath along with the axillary vein and brachial plexus. The second part lies deep to the pectoralis minor muscle and the third part lies between the inferolateral border of the pectoralis minor and the inferior border of the teres major muscle.[1]

The axillary artery usually gives off six branches. The first part of the artery gives superior thoracic artery. The second part gives lateral thoracic and thoracoacromial branches. The third part gives subscapular artery, anterior and posterior circumflex humeral artery.[2]

There is extensive collateral circulation associated with the branches of subclavian and axillary arteries particularly around scapula so that the sound knowledge of neuromuscular variation is important for surgeons who remove the axillary lymph nodes, to anaesthesiologist and orthopaedic surgeons considering the frequency of procedures done in this region. It is very common to find the variations in the branching pattern. sometimes many of the branches may originate from a common stem which is known as the clumping of branches.[3]

\section{MATERIALS AND Methods}

During routine dissection in the department of anatomy, V.S.S medical college, Burla, axillary arteries belonging to 60 upper limbs of 30 cadavers(10 females and 20 males) were observed during 2008-2011.Out of them 30 were of right side and 30 were of left side. These limbs were dissected retaining the continuity with the trunk. The axillary region was exposed and the branching pattern was observed.

\section{III.}

RESULTS

During routine dissection of under graduate students we came across 3 unilateral cases of clumping of braches of axillary artery out of sixty upper limb specimen of 30 cadavers. In all the 3 cadavers the abnormal branching pattern was observed on the right side axilla. But the branching pattern was normal on the left side of the same cadavers.

The observations were tabulated(table 1) to study the variations.

\section{DISCUSSION}

Vascular variations are the commonest anatomical variations .most of the time they do not cause any symptoms or harm to the subject. During embryogenesis the lateral branch of seventh cervical inter segmental artery becomes enlarged to form the axial artery of upper limb which on further development becomes axillary, branchial, its bud gives rise to radial and ulnar arteries[4]. The arterial anomalies in the upper limb are due to defects in embryonic development of the vascular plexus if the upper limb bud. This may be due to arrest at any stage of development of the vascular plexus showing regression, retension or reappearance and may lead to variations in the arterial origins and courses of the major upper limb vessels[5] 
The axillary artery is usually described as giving off six branches although the number varies because two or more arteries often arise together instead of separately or two branches of an artery arise separately instead of form the usual common trunk. Thus instead of six there may be 5-11 branches. A rare but striking anomaly of the axillary artery is for it to divide into two branches that proceed down the arm. When this occurs, usually one of the arterial stems in the arm runs more superficially than the other, and they are therefore sometimes distinguished as brachial artery and superficial brachial artery. This apparent doubling of the brachial artery more commonly occurs in the arm than in the axilla [6].

The anomalies can be explained by the persistence of embryological vessels. Genetic influences are deemed to be prevalent causes of such variation, although factors like fetal position in utero, first limb movement or unusual musculature cannot be excluded[7].

The first part of the axillary artery may also provide an accessory thoraco-acromial artery. The third part of the axillary artery is occasionally covered by a muscular slip (an axillary arch muscle) derived from the upper part of the tendon of latissimus dorsi. Unusual branches of the axillary artery include a glandular artery to lymph nodes and skin of the axilla (so called alar thoracic artery) and an accessory lateral thoracic artery[8].In our study we have found alar thoracic artery(fig 2 ) which is supplying axillary lymph nodes and one accessory lateral thoracic arising from the main lateral thoracic and supplying serratus anterior(fig 3 ).

Saeed and his co-workers have explained similar variation, where a common trunk from $2^{\text {nd }}$ part of the axillary artey gave rise to lateral thoracic, circumflex humeral, subscapular and thoraco dorsal arteries[[9].However in our observation in one case we have found a common trunk giving rise to lateral thoracic artery,thoracodorsal and circumflex scapular and the lateral thoracic gives rise to one accessory lateral thoracic artery which is not reported till date (fig 3 ).In another case we have reported the second part giving rise to a common trunk for thoraco acromial, lateral thoracic, subscapular, alar thoracic, circumflex scapular and posterior circumflex humeral(fig 2 ).

The branches of the third part are subject to great variations. The two circumflex arteries may arise from acommon trunk, usually alone or rarely together with profunda brachii and muscular braches. Very rarely it may give rise to a common trunk, from which may arise the subscapular, anterior and posterior circumflex humeral, profunda brachii and ulnar collateral arteries[9].In our cases also we have found most of the branches are coming from $2^{\text {nd }}$ part not from the $3^{\text {rd }}$ part.

\section{CONCLUSION}

The increasing use of invasive diagnostic and interventional procedures in cardiovascular diseases makes it important that the type and frequency of vascular variations are well documented and understood. Knowledge of variation is important during bypass surgery between axillary and subclavian artery in case of subclavian artery occlusion, while treating the aneurysm of axillary artery,during coronary bypass and flaps in reconstructive surgeries, during catheterization or cannulation of axillary arteries for various purposes[10]

Anomalies in the origin and course of principal arteries are having practical importancefor the vascular radiologist. Chronic dislocation of the shoulder joint the incision is transverse and it may injure the branches of axillary artery[11]. So during surgery the abnormal branch may be a definite cause of bleeding if its presence is not kept in mind[12].

Sound knowledge of axillary artery variation is important for surgeons, since except for the popliteal, the axillary artery is more frequently lacerated by violence than any other surgery. It has been ruptured in attempt to reduce old dislocations, especially when the artery is adherent to the articular capsule[13].For these clinical applications the knowledge.

VI. Tables And Figures

TABLE 1 SHOWING CLUMPING OF BRANCHES OF AXILLARY ARTERY

\begin{tabular}{|l|l|l|l|}
\hline Branches from & Case-1(fig 1) & Case-2(fig 2) & Case-3(fig 3) \\
\hline First part & Superior thoracic & Superior thoracic & Superior thoracic \\
\hline Second part & 1.Thoracoacromial & Common trunk for & Common trunk branching into \\
& 2.common trunk for & -Thoraco- acromial & -Thoraco- dorsal \\
& - Lateral Thoracic & -Lateral thoracic & caleral thoracic \\
& - Sub scapular & -Alar thoracic & -Sub scapular \\
& - Posterior circumflex humeral & -Post circumflex humeral & \\
\hline Third part & Ant. Circumflex humeral & Ant. Circumflex humeral & $\begin{array}{l}\text { Ant. Circumflex humeral } \\
\text { Post circumflex humeral }\end{array}$ \\
\hline
\end{tabular}




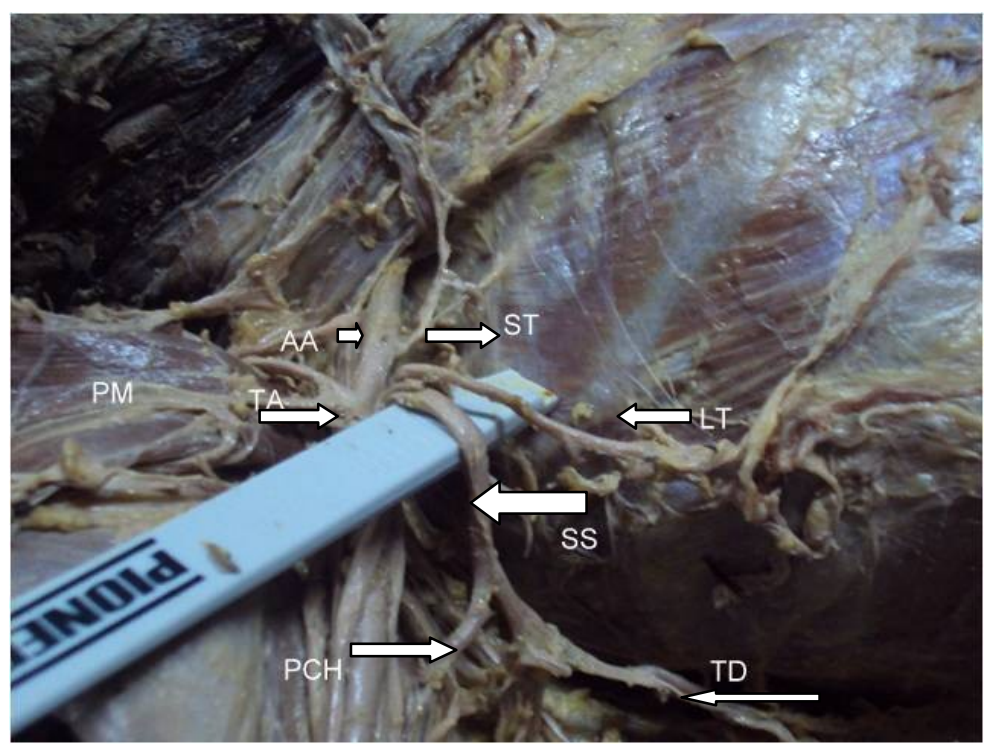

Figure 1:-2nd part of axillary artery showing clumping where the common trunk giving rise to lateral thoracic subscapular and posterior circumflex humeral.(AA-axillary artery, ST-superior thoracic, TA-thoracoacromial, PM-pectoralis minor, LTlateral thoracic, SS-subscapular, PCH-posterior circumflex humeral, TD-thoracodorsal)

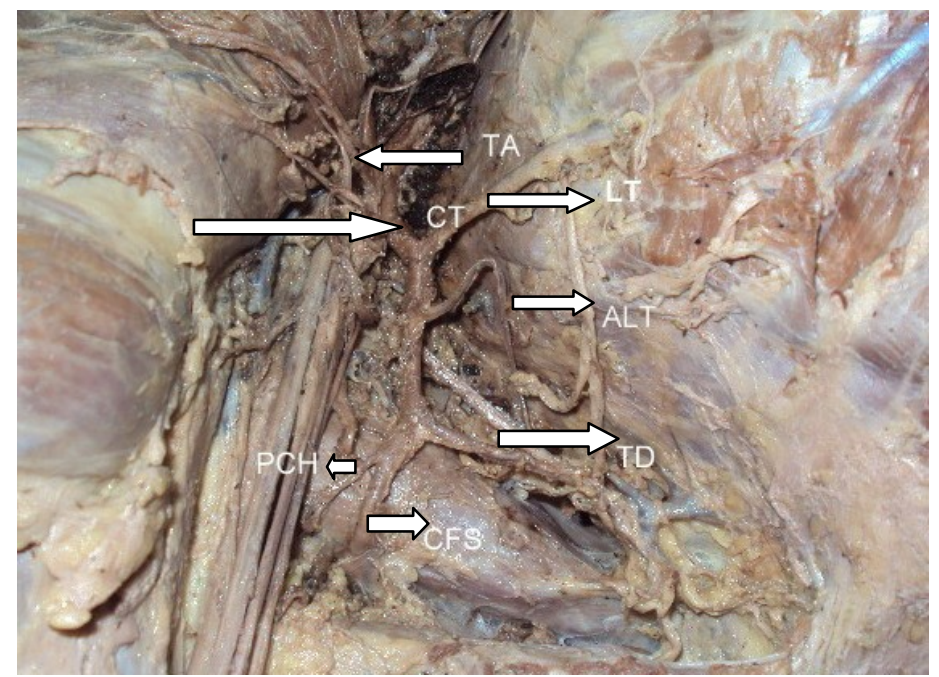

Figure 2:- $2^{\text {nd }}$ part of axillary artery showing a common trunk for thoracoacromial, lateral thoracic, alar thoracic, subscapular, posterior circumflex scapular, circumflex scapular.(TA-thoracoacromial, CT-common trunk, LTlateral thoracic, ALT-alar thoracic, PCH-posterior circumflex humeral, CFS-circumflex scapular)

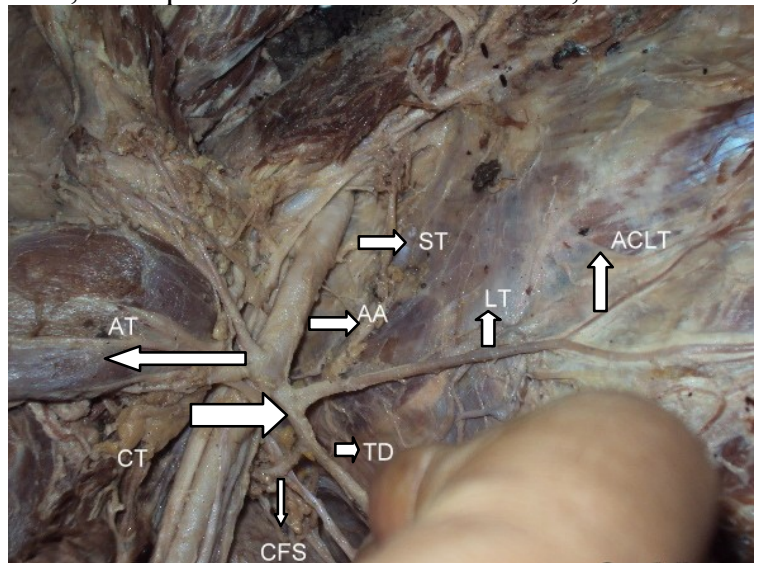

Figure 3; $2^{\text {nd }}$ part of axillary artery showing common trunk for lateral thoracic, thoracodorsal and circumflex scapular( ST-superior thoracic, AT-acromiothoracic, AA-axillary artery,CT-common trunk, LT-lateral thoracic, ACLT-accessory lateral thoracic, CFS-circumflex scapular ) 


\section{REFERENCES}

[1] Standring S editor. Gray's Anatomy: The Anatomical Basis of Clinical Practice. 40th ed.2008. Churchill-Livingstone: Elsevier. ISBN978-0-443-06684-89.

[2] Snell R. Clinical Anatomy for medical students. $4^{\text {th }}$ ed. Boston; Little Brown Co.; 1992.

[3] Hollinshed WH. Anatomy for surgeons in general surgery of upper limb. The back and limbs. A Heber Harper BookBook, New York, 1958.pp- 290-300

[4] Jurjus AR, Correa-De-Aruaujo R, Bohn RC.Bi l a t e r a l d o u b l e a $x$ i l i a r y a $r$ t e y :embryological basis and clinical implications. Clin Anat 1999; 12: 135-140.

[5] Hamilton WJ, Mossman HW. Cardiovascular system. In: Human embryology. 4th ed. Baltimore: Williams and Wilkins, 1972: 271290.)

[6] Hollinshead, W. H. \& Rosse, C. Text Book of Anatomy. $4^{\text {th }}$ Ed. Harper \& Row, Philadelphia, 1985. pp.187-189.

[7] M. Rodriguez-Niedenfuhr, G. J. Burton, et al.,Development of the arterial pattern in the upper limb of staged human embryos: normal development and anatomic variations. J. Anat. 2001; 199: 407-41

[8] Ronald A. Bergman, Adel K. Afifi, , Ryosuke Miyauchi. Axillary Artery Opus II: Arteries. Cardiovascular System . Illustrated Encyclopedia of Human Anatomic Variation:2006

[9] Saeed M,Rufai AA,Elsayed SE,Sadiq MS. Variations in the subclavian-axillary arterial system.2002;23(2):206-212.

[10] Charitou A,AthanasiouT,Morgan IS,Del Stanbridge R.Use of Cough Lok can predispose to axillary artery thrombosis after a Robicsek procedure.Interact Cardiovasc Thorac Surg 2003; 2(1):68-69

[11] Shoulder joint. In: Decker GAG, du plessis DJ. Lee Mc Gregor's Synopsis of Surgical Anatomy. 12th ed. Mumbai: K.M. Varghese company, 1986: 451

[12] Cervicobrachial region. In: Samuel L Turek's orthopaedics: Principles and their Applications: Vol 2. 4th ed. New Delhi: Jaypee brothers, 1989:913.

[13] Rohini R.Karambelkar ,Avinash D Shewale , Umarji .B.N. Variations in Branching Pattern of Axillary Artery and its Clinical Significance,Anatomica Karnataka,5(2),2011,47-51 